\title{
Visible two-dimensional photonic crystal slab laser
}

\author{
Zhaoyu Zhang, Tomoyuki Yoshie, ${ }^{a}$ Xiaoliang Zhu, Jiajing Xu, and Axel Scherer ${ }^{\text {b) }}$ \\ Department of Electrical Engineering, California Institute of Technology, Pasadena, California 91125
}

(Received 31 May 2006; accepted 8 July 2006; published online 14 August 2006)

\begin{abstract}
The authors describe the fabrication and performance of photonic crystal lasers fabricated within thin membranes of InGaP/InGaAlP quantum well material and emitting in the visible wavelength range. These lasers have ultrasmall mode volumes, emit red light, and exhibit low threshold powers. They can be lithographically tuned from 650 to $690 \mathrm{~nm}$. Their cavity volumes of approximately $0.01 \mathrm{\mu m}^{3}$ are ideally suited for use as spectroscopic sources. (C) 2006 American Institute of Physics. [DOI: 10.1063/1.2336721]
\end{abstract}

Photonic crystal cavities offer many advantages over more conventional cavities for achieving ultrasmall modal volumes while maintaining high quality factors. ${ }^{1-5}$ When combining such cavities with light emitting active materials, such as quantum wells ${ }^{1}$ and quantum dots, ${ }^{3}$ it is possible to form ultrasmall lasers that can be integrated in dense arrays, and in which each laser cavity supports only a very few optical modes. This results in the potential for high frequency modulation of such lasers, ${ }^{6}$ which has made these devices particularly interesting for applications in optical data communication. Therefore, most research on photonic crystal lasers has thus far focused on near-infrared wavelength emission using InGaAsP or InGaAs active materials. Although some research groups have started to explore the visible wavelength range, ${ }^{,-10}$ it has been difficult to obtain small mode volume lasers in visible light emitting material systems due to high surface carrier recombination velocities or the lack of high refractive index contrast substrates for light confinement in the vertical direction. Visible photonic crystal lasers operating in the spectral vicinity of $670 \mathrm{~nm}$ could enable a broad range of important applications, including high density optical recording, high resolution visible laser projection displays, and, most importantly, compact spectroscopic sources as ultrasmall sensors for biological and chemical detections within small sample volumes. In this letter, we present our preliminary experimental results of such a two-dimensional photonic crystal slab laser that can satisfy these needs.

Photonic crystal slab structures were first grown by metal organic chemical vapor deposition of InGaP/InGaAlP quantum well material on top of sacrificial AlGaAs layers supported by GaAs substrates. Optical gain was provided by two $7 \mathrm{~nm}$ thick and compressively strained InGaP quantum wells which were separated by $10 \mathrm{~nm}$ InGaAlP barriers [Fig. 1(a)]. The quantum well active material was placed in the center of a $170 \mathrm{~nm}$ thick InGaAlP slab, with a $700 \mathrm{~nm}$ thick sacrificial AlGaAs layer between the slab and the GaAs substrate. The active quaternary material was designed to emit light at around $670 \mathrm{~nm}$ [Fig. 1(b)]. From the compressive strained quantum wells, light was strongly coupled into transverse electric modes. This epitaxially grown material was coated with a $100 \mathrm{~nm}$ SiON hard mask and $200 \mathrm{~nm}$ of Zep520 electron beam resist.

\footnotetext{
${ }^{a}$ Present address: Department of Electrical and Computer Engineering, Duke University, Durham, NC 27708-0291.

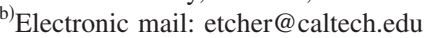

Electron beam lithography was then used to define the photonic crystal cavity pattern within the Zep520 resist. Reactive ion etching (RIE) was subsequently used to transfer the pattern from that resist into the SiON etch mask by using a $\mathrm{CHF}_{3}$ plasma. After removal of the resist, the hard mask pattern was further transferred through the active layer with an iodine-based inductively coupled plasma RIE. Time controlled oxidation of the AlGaAs by water vapor followed by the potassium hydroxide $(\mathrm{KOH})$ chemical dissolution of the aluminum oxide formed the suspended slab membranes, as shown in Fig. 2. Finally, diluted buffered hydrofluoric acid was used to remove the SiON etch mask.

The suspended photonic crystal slab cavities were optically pumped at room temperature using $5 \mathrm{~ns}$ pulses at $10 \mathrm{kHz}(0.005 \%$ duty cycle) with a $408 \mathrm{~nm}$ InGaN semiconductor diode laser. The pump beam was focused onto the sample surface with a $50 \times$ objective lens to form an excitation beam spot size about $2 \mu \mathrm{m}$ in diameter. The excitation power used in this letter was determined by dividing the averaged pulse power by the duty cycle. The emission from the lasers was then collected through the same lens and their spectra detected with a liquid nitrogen cooled chargecoupled device (CCD) (Princeton Instruments, Spec10) detector filtered by a monochromator (Acton, SpectraPro). The monochromator entrance slit width was set to $10 \mu \mathrm{m}$ and the 1200 groove $/ \mathrm{mm}$ grating was used, resulting in a spectral resolution of approximately $0.1 \mathrm{~nm}$. An additional flip-up mirror was used to guide the light into a CCD imaging system to view the near-field images of the lasers as well as the

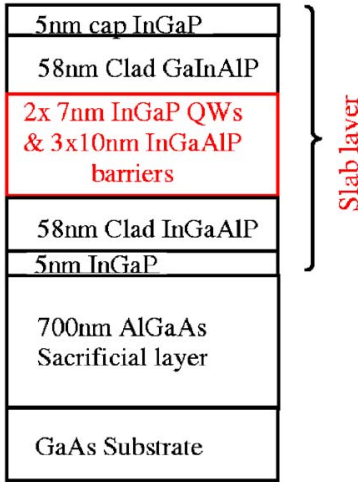

(a)

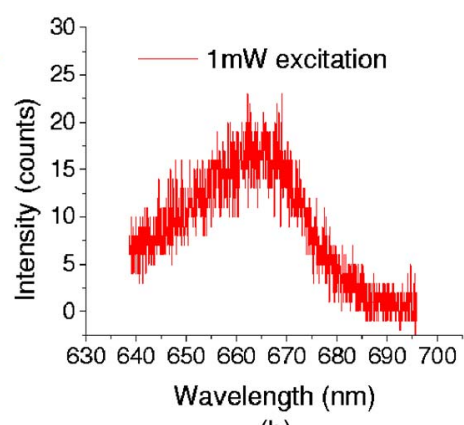

(b)
FIG. 1. (Color online) (a) Schematic epitaxial layer sequence of our slab composition. (b) A typical photoluminescence emission spectrum of the grown wafer. 


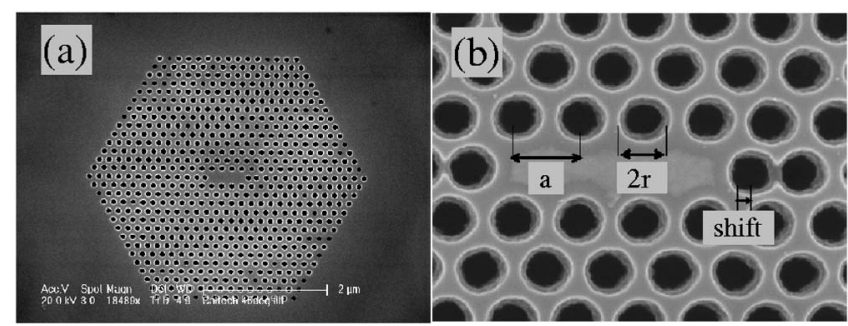

FIG. 2. Scanning electron microscopy micrographs of photonic crystal laser cavity. (a) Low magnification image showing the entire device and (b) higher resolution image showing the details of the cavity.

excitation laser spot. The imaging optics also permitted the observation of laser cavity modes.

For this work, we used a high $Q$ cavity design proposed by Akahane et al..$^{5}$ consisting of a $L 3$ linear defect (removing three holes) within a triangular photonic crystal lattice of holes on a thin high-index slab. This design reduces the loss in the vertical direction by shifting the holes at the end of the defect area outwards. Figure 2 shows the top view of a fabricated photonic crystal microcavity slab. The lattice spacing, $a$, the radius of hole, $r$, and the shift of the hole were defined, as shown in Fig. 2(b). We lithographically controlled the ratio between the hole shift and the lattice spacing (shift/a) to be 0.2 . Also, we lithographically varied the lattice spacing (a) within a range from 0.14 to $0.18 \mu \mathrm{m}$ and the ratio between the hole radius and lattice spacing $(r / a$, the porosity factor) from 0.25 to 0.29 . Fourteen periods of photonic crystal lattice [Fig. 2(a)] were used to surround the defect within the $170 \mathrm{~nm}$ thick quantum well active layer slab which has a refractive index of around 3.4 at $670 \mathrm{~nm}$. The photonic crystal and cavity dimensions were designed to match the $670 \mathrm{~nm}$ emission wavelength of the $\mathrm{InGaP}$ active material. Figure 3 shows luminescence spectra and the $L$ (excitation power)- $L$ (lasing peak intensity) curve from a device with lattice parameter $a=170 \mathrm{~nm}$ and porosity factor $r / a=0.26$. The laser threshold was determined to be approximately $250 \mu \mathrm{W}$, and the linewidth was measured as $0.3 \mathrm{~nm}$ at threshold, yielding an effective $Q$ of about 2000. Below threshold, only a broad background from the gain medium was measured from this cavity. Above $450 \mu \mathrm{W}$, heating of the laser cavity limited the output power and the $L-L$ curve saturated. The two-dimensional (2D) photonic crystal cavity peak can be tuned by changing either the porosity or the lattice parameter of the photonic crystal lattice surrounding the nanocavity. Figure 4 shows a laser with a slightly lower porosity (porosity factor $r / a=0.25$ ). For this laser, the measured laser threshold was $400 \mu \mathrm{W}$ and the device again saturated as a result of cavity heating above $1 \mathrm{~mW}$. Both cavities
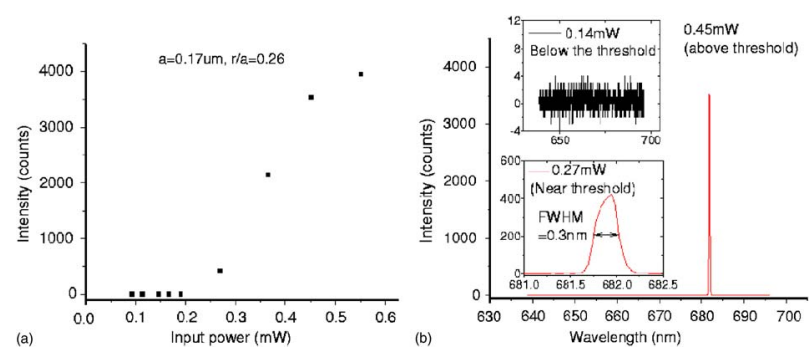

FIG. 3. (Color online) (a) $L-L$ curve and (b) typical lasing spectrum of an InGaP 2D photonic crystal laser with a lattice parameter of $a=0.17 \mu \mathrm{m}$ and porosity factor $r / a=0.26$.

Downloaded 08 Sep 2006 to 131.215.225.158. Redistribution subject to AIP license or copyright, see http://apl.aip.org/apl/copyright.jsp
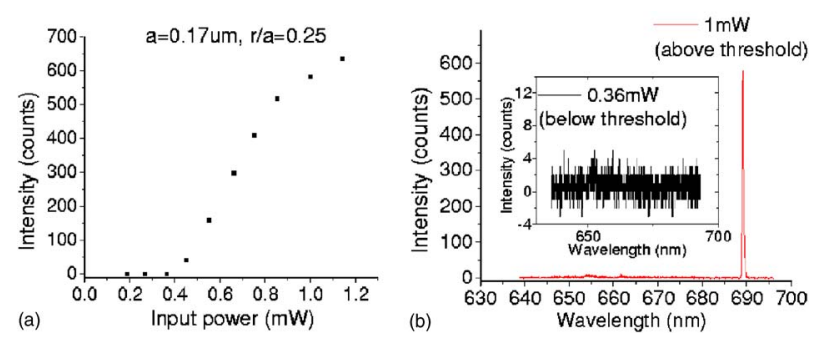

FIG. 4. (Color online) (a) $L-L$ curve and (b) typical lasing spectrum for an InGaP 2D photonic crystal laser with a lattice parameter of $a=0.17 \mu \mathrm{m}$ and porosity factor $r / a=0.25$.

exhibited distinct threshold and linearity in the output power above threshold but before saturation. The lasing characteristic was very sensitive to the position of the excitation beam spot $(\sim 1 \mu \mathrm{m}$ movement), which indicates that lasing occurs from a localized defect mode. Many of the photonic crystal cavity geometries that we defined did not result in demonstration of lasing, and we show a typical spectrum result from a nonlasing cavity in Fig. 5, along with the $L-L$ curve. linear increase of emission with increasing excitation power was observed, saturating again at high excitation powers due to heating of the cavity. No lasing peak was observed, and the Fabry-Pérot filtered luminescence emission was not bright enough to observe with our experimental spectroscopic measurement setup.

Figure 6(a) shows the influence of changing of the porosity on the laser emission wavelength, whereas Fig. 6(b) shows a similar tuning curve for changes in the photonic crystal lattice parameter. The uneven spectral shifts result from fabrication variation and device scaling only in two rather than three dimensions due to the fixed slab thickness. These data indicate that the InGaP photonic crystal lasers can be lithographically tuned throughout the gain emission of the quantum wells. The accuracy of such tuning is only limited by the quality and reproducibility of the fabrication process. It should be noted that the minimum feature size required for the successful definition of the photonic crystal mirror surrounding the laser cavities is approximately $80 \mathrm{~nm}$, which is the distance between two adjacent holes in the slab. Compared to conventional InGaAsP material systems operating at $1550 \mathrm{~nm}$, our fabrication process has to overcome the feature size scaling associated with the 2.3 times decreases in wavelength. Nevertheless, with our fabrication approach, it is possible to define dense arrays of laser cavities that are separated by as little as $2 \mu \mathrm{m}$. Compact multispectral sources can therefore be defined within the visible wavelength range.

The use of photonic crystal lasers for spectroscopic analysis has been described earlier, ${ }^{11}$ and offers the opportu-
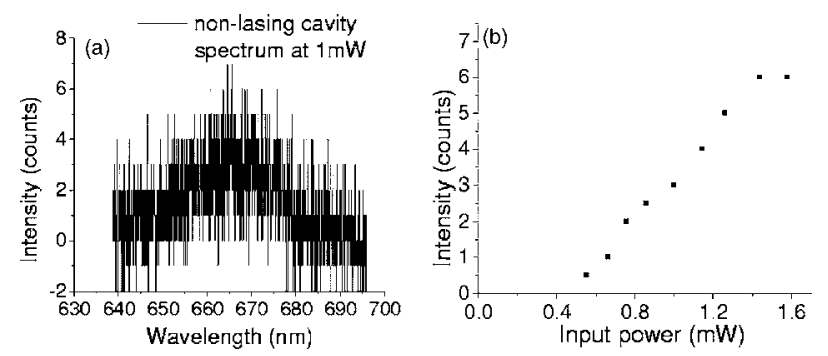

FIG. 5. (a) Spectrum and (b) $L-L$ curve of a typical nonlasing photonic crystal cavity. In this case, no inflection was measured and a more or less 

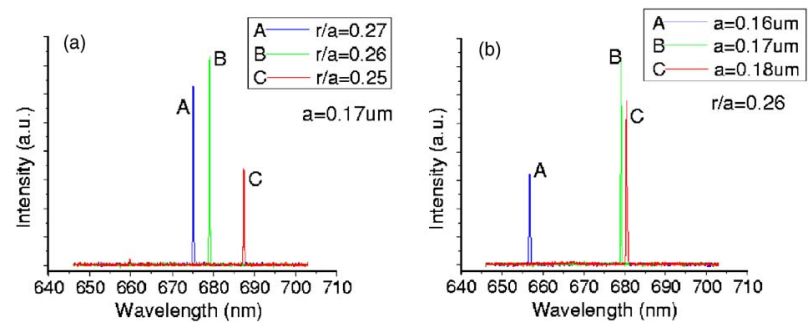

FIG. 6. (Color online) Spectra of (a) lattice spacing fixed to $0.17 \mu \mathrm{m}$ and (b) $r / a$ fixed to 0.26 (the uneven spectral shifts result from fabrication variation and device scaling only in two dimensions due to the fixed slab thickness).

nity to define ultrasmall optical cavities with enormous optical field intensities. These devices have been used in the past as refractive index sensors, indicating the refractive index of volumes as small as $10^{-17}$ liters, limited by the mode volume of $0.03 \mu \mathrm{m}^{3}$ at $1550 \mathrm{~nm}$ wavelength. In our experiments, we have defined lasers within a wavelength range that is even more interesting for spectroscopic applications, as many of the fluorophores used for biological analysis are limited to the visible spectrum, and single-photon detectors are available at such wavelengths. Moreover, these devices may become very interesting sources for Raman spectroscopy and other specific measurements of the chemical composition of the femtoliter contents of an optical nanocavity. We expect InGaP lasers to be very useful for biochemical analysis as well as for efficient displays and high frequency lasers in the near future.
The authors would like to thank EpiWorks Inc. for wafer growth, Yueming Qiu from Jet Propulsion Laboratory, and Koichi Okamoto and Terrell Neal for the generous help in the fabrication and measurement setup. This work was supported by the AFOSR under Contract No. F49620-03-10418, by Boeing Corp. under the SRDMA program, and by Intel Corp.

${ }^{1}$ O. Painter, R. K. Lee, A. Scherer, A. Yariv, J. D. O’Brien, P. D. Dapkus, and I. Kim, Science 284, 1819 (1999).

${ }^{2}$ M. Loncar, T. Yoshie, A. Scherer, P. Gogna, and Y. M. Qiu, Appl. Phys. Lett. 81, 2680 (2002).

${ }^{3}$ T. Yoshie, O. B. Shchekin, H. Chen, D. G. Deppe, and A. Scherer, Electron. Lett. 38, 967 (2002).

${ }^{4}$ K. J. Vahala, Nature (London) 424, 839 (2003).

${ }^{5}$ Y. Akahane, T. Asano, B. S. Song, and S. Noda, Nature (London) 425, 944 (2003).

${ }^{6}$ T. Yoshie, M. Loncar, A. Scherer, and Y. Qiu, Appl. Phys. Lett. 84, 3543 (2004).

${ }^{7}$ M. Campbell, D. N. Sharp, M. T. Harrison, R. G. Denning, and A. J. Turberfield, Nature (London) 404, 53 (2000).

${ }^{8}$ X. Wu, A. Yamilov, X. Liu, S. Li, V. P. Dravid, R. P. H. Chang, and H. Cao, Appl. Phys. Lett. 85, 3657 (2004).

${ }^{9}$ Y. S. Choi, K. Hennessy, R. Sharma, E. Haberer, Y. Gao, S. P. DenBaars, S. Nakamura, E. L. Hu, and C. Meier, Appl. Phys. Lett. 87, 243101 (2005).

${ }^{10}$ C. Meier, K. Hennessy, E. D. Haberer, R. Sharma, Y. S. Choi, K. McGroddy, S. Keller, S. P. DenBaars, S. Nakamura, and E. L. Hu, Appl. Phys. Lett. 88, 0331111 (2006).

${ }^{11}$ M. Loncar, A. Scherer, and Y. M. Qiu, Appl. Phys. Lett. 82, 4648 (2003). 